\title{
Strates
}

STRATES Matériaux pour la recherche en sciences sociales

$11 \mid 2004$

Jeune recherche, la vitalité d'un laboratoire

\section{Mettre en technique : conseillers agricoles et pollution de l'eau en Bretagne}

\section{Hélène Brives}

\section{(2) OpenEdition \\ 1 Journals}

Édition électronique

URL : http://journals.openedition.org/strates/440

DOI : $10.4000 /$ strates. 440

ISSN : $1777-5442$

Éditeur

Laboratoire Ladyss

Édition imprimée

Date de publication : 1 janvier 2004

ISSN : 0768-8067

Référence électronique

Hélène Brives, « Mettre en technique : conseillers agricoles et pollution de l'eau en Bretagne », Strates [En ligne], 11 | 2004, mis en ligne le 14 janvier 2005, consulté le 08 septembre 2020. URL : http:// journals.openedition.org/strates/440 ; DOI : https://doi.org/10.4000/strates.440

Ce document a été généré automatiquement le 8 septembre 2020

Tous droits réservés 


\title{
Mettre en technique : conseillers agricoles et pollution de l'eau en Bretagne
}

\author{
Hélène Brives
}

1 Thèse de sociologie soutenue à l'université de Paris X, le 29 octobre 2001, sous la direction de Nicole Eizner, directeur de recherche au laboratoire Ladyss/Cnrs (356 p.)

Dans son édition du jeudi 29 juillet 1999, Le Monde, titrait : « La Bretagne échoue dans sa 'reconquête' de l'eau ».

On pouvait lire en sous-titre : « Malgré la mise en place d'un programme de 1,4 milliard de francs, la pollution des sols et des rivières par les nitrates d'origine agricole dans les quatre départements de la région est remontée, en 1998, à ses niveaux records ».

Plus loin dans le corps de l'article, un responsable du programme incriminé admettait : «On peut tout à fait affirmer que 1,4 milliard de francs sont dépensés et que la qualité de l'eau ne s'améliore pas. Mais on ne peut pas dire qu'il ne se passe rien. » Si l'expert interviewé souhaitait avant tout justifier de son travail, il faisait également valoir une nuance d'importance : l'intérêt des opérations de lutte contre la pollution de l'eau par les nitrates d'origine agricole ne pourrait être mesuré seulement à l'aune des taux des nitrates dans l'eau. Des choses, bien qu'importantes, échapperaient donc à la mesure.

5 Les conseillers agricoles, salariés des Chambres départementales d'Agriculture travaillant quotidiennement auprès des agriculteurs, sont confrontés à ce problème de pollution qui, en Bretagne sans doute plus qu'ailleurs, représente un enjeu économique, technique et social considérable. Que font-ils? De quelles façons abordent-ils le problème?

6 C'est tout d'abord cette interrogation qui a animé notre recherche et qui demandait d'aller voir concrètement ce qu'était l'activité quotidienne des conseillers agricoles confrontés à ce problème de pollution.

7 La pollution de l'eau met en effet sur la sellette la définition de l'agriculture moderne et ses modèles de production autour desquels le groupe professionnel des conseillers 
agricoles s'est constitué aux débuts des années soixante. L'existence, en France, de ces professionnels, ne peut être dissociée du mouvement de modernisation de l'agriculture entamé aux lendemains de la Seconde Guerre mondiale.L'ambition politique de l'époque vise à une complète réorganisation du secteur agricole et de ses producteurs, et désigne, dans cette perspective, les façons de produire et les «façons d'être agriculteurs » adéquates (Rémy 1981). Un imposant appareil d'encadrement du secteur se met en place, sous le nom de "Vulgarisation " puis de "Développement », et ses agents sont chargés de promouvoir ces modèles de l'agriculture moderne. bretons. La Bretagne a bâti sa richesse sur une des plus grandes concentrations européennes d'élevages intensifs associés aux industries d'approvisionnement et de transformation. Cependant, après avoir permis de transformer en quelques années la France d'après-guerre en premier pays européen pour les exportations agricoles, ces modèles de production, qui n'intéressaient que quelques poignées de spécialistes il y a encore quelques années, posent aujourd'hui problème et sont publiquement débattus jusque dans les colonnes d'un quotidien généraliste comme Le Monde.

Les recherches qui traitent directement des conseillers agricoles sont rares, mais elles s'insèrent dans le vaste ensemble de travaux de la sociologie rurale et de l'agriculture qui, s'intéressant aux transformations du secteur agricole, rencontrent cette catégorie particulière d'acteurs, figure emblématique de l'appareil d'encadrement de l'agriculture. Rouage d'un dispositif qui les dépasse, l'activité de ces techniciens apparaît le plus souvent secondaire au regard d'une préoccupation majeure de la sociologie, celle de l'évolution des rapports sociaux liée à l'organisation de la production agricole. Ces études portent soit sur le processus d'innovation lui-même (comment il se produit, se propage, ce qui lui résiste), soit sur les modèles politiques que sous-tendent les modèles techniques de l'agriculture moderne, pour en dénoncer les mécanismes de domination.

Les analyses sociologiques de la profession de conseiller agricole menées par Jacques Rémy ou par Bruno Lémery mettent en évidence que la définition de ce qui est "technique", constitue un enjeu majeur de la définition d'une compétence de conseiller agricole (Rémy, 1977, 1984 ; Lémery, 1991). Ces travaux montrent comment la définition de la technique recouvre des relations sociales, des conflits entre les conseillers et leurs employeurs, des enjeux de reconnaissance professionnelle, des modalités d'intervention auprès des agriculteurs. À une époque où les modèles de l'agriculture moderne laissent toujours plus d'agriculteurs sur le bord du chemin, ces recherches problématisent la place de la technique dans les rapports sociaux seulement dans ses conséquences sociales négatives.

11 Les conseillers bretons que nous avons suivis dans leur travail sont aujourd'hui confrontés quotidiennement au problème de la pollution de l'eau. Il importe donc à présent d'interroger la technique également dans le traitement de la nature qu'elle opère et de s'intéresser aux objets concrets de la pollution et de son traitement par les conseillers (Mormon, 1993).

12 C'est pourquoi il nous a semblé important de faire l'hypothèse que c'est dans leur pratique ordinaire que les conseillers construisent ce qu'est la technique. Pour cela, il a fallu interroger la catégorie de la technique, dans les discours, mais aussi dans les pratiques concrètes, sans en donner de définition a priori, et en se séparant de ce que Nicolas Dodier appelle les « visions inquiètes » qui font référence à un modèle global et 
univoque de changement technique (Dodier, 1995). Ce travail a été ainsi l'occasion d'élaborer un cadre d'analyse basé sur une approche pragmatique de l'action, c'est-àdire de partir de l'activité concrète des conseillers pour comprendre ce qu'est la technique et ce qu'elle fait. Dans une telle perspective, l'enquête ethnographique s'est imposée comme un outil privilégié.

Le compte rendu de ce travail s'appuie sur la mise en perspective de cinq « segments d'activité » des conseillers agricoles qui sont autant d'histoires des conseillers et de la pollution de l'eau : une histoire de référence retraçant l'installation de la question de la pollution dans l'activité des conseillers autour des années soixante-dix, une réunion d'un groupe de développement sur le thème de la fertilisation du maïs, une démonstration d'épandage de lisier, les débuts d'une opération bassin versant et la construction collective d'une carte des terres épandables à l'échelle d'une commune.

Une approche pragmatique conduit à appréhender ces histoires comme des énigmes à résoudre et à considérer l'action comme fait premier, c'est-à-dire à analyser non pas l'interaction entre des entités pré-établies, mais bien la construction de ces entités dans le processus même de l'interaction. Les institutions et autres formes de cadrages de l'action sont comprises comme le produit des activités sociales ordinaires que solidifie le travail de l'histoire et qui ne tiennent que si elles sont réactivées dans les pratiques ordinaires, dans l'action. À travers ce processus «d'institutionnalisation», on saisit comment les activités sociales ordinaires participent à la structuration du cadre des interactions ultérieures (Berger, Luckmann, 1996).

Ce travail a permis de comprendre comment les conseillers organisent leur mode d'action en sélectionnant les entités (acteurs, connaissances...) qui peuvent y figurer et celles qui en sont exclues, pour tenter à la fois de suivre le problème de la pollution dans ses développements les plus inattendus et en même temps, d'en limiter les débordements.

Leurs efforts de cadrage s'organisent autour de la catégorie de technique, qui délimite et ordonne le collectif associé au travail des conseillers. À travers la construction de la catégorie de technique, toujours mouvante, se redéfinit en permanence le champ de compétence de conseiller agricole. Cet effort d'agencement, de mise en ordre, que les conseillers mènent dans le cours même de leur activité ordinaire, peut être qualifié de "mise en technique», comme Bruno Latour parle de mise en nature et de mise en société (Latour). La frontière entre un ordre technique et un ordre social ou politique que les conseillers travaillent perpétuellement à établir, ne reprend pas le "grand partage » entre nature et société que dénonce Latour, mais dessine un espace au sein duquel leur activité est possible. En se centrant sur la technique, les conseillers n'excluent pas pour autant d'intervenir sur l'organisation sociale du monde agricole.

17 L'intérêt de l'approche de sociologie pragmatique élaborée dans le cadre de cette thèse a été d'établir concrètement le lien entre des processus de mobilisation/production de connaissances et des formes d'organisation des relations au sein d'un collectif. L'analyse de situations d'action conjointe entre agriculteurs et conseillers agricoles sur la pollution de l'eau a montré comment la production de connaissances opératoires pour l'action et la mobilisation des acteurs concernés constituent un seul et même processus. Le choix des connaissances mobilisées sélectionne les acteurs autorisés à intervenir et organise les relations entre eux. Dans le cours de l'action sont produits en même temps des connaissances sur la nature et des rapports sociaux ; des vérités et des identités sont établies. On peut alors comprendre par quels mécanismes concrets une 
loi est appliquée, un modèle de production imposé, un agriculteur convaincu de changer ses pratiques, une norme produite. Les lois et les sciences peuvent être pensées ensemble comme les deux versants d'un seul et même processus d'organisation de la vie collective.

\section{BIBLIOGRAPHIE}

Berger P., Luckmann T., 1996, La construction sociale de la réalité, Paris, A. Colin, (première édition américaine : 1966).

Dodier N., 1995, Les hommes et les machines. La conscience collective dans les sociétés technicisées, Paris, Métailié.

Latour B., 1997, Nous n'avons jamais été modernes. Essai d'anthropologie symétrique, Paris, La Découverte.

Lemery B., 1991, Lectures sociologiques des activités de conseil technique en agriculture. Essai sur les processus de rationalisation, thèse de doctorat de sociologie, Université Lumière/Lyon, 2 volumes.

Mormont M., 1993, Sciences sociales et environnement. Approches et conceptualisations, rapport au ministère de l'Environnement, Belgique, Fondation Universitaire Luxembourgeoise.

Remy J., 1977, « Le 'malaise des conseillers' ou l'histoire d'une institution pédagogique », Éducation Permanente, no 37.

Remy J., 1981, Le métier d'agriculteur. Façons de produire et façons d'être des agriculteurs sarthois, Paris, INRA, 3 tomes

Remy J., 1984, « Les conseillers agricoles devant le développement », Pour, no 93.

\section{INDEX}

Keywords : Bretagne

Mots-clés : Eau 